\title{
Automatic detection of lung nodules in CT datasets based on stable 3D mass-spring models
}

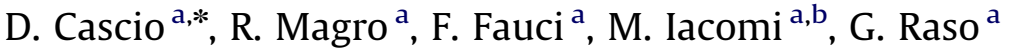 \\ a Dipartimento di Fisica, Università degli Studi di Palermo, Italy \\ ${ }^{\mathrm{b}}$ Institutul de Ştiinţe Spaţiale, P.O. Box MG-23, Ro 077125, Bucharest-Măgurele, Romania
}

\section{A R T I C L E I N F O}

\section{Article history:}

Received 15 February 2011

Accepted 4 September 2012

\section{Keywords:}

Segmentation

Lung nodules

Active contours models

Computer tomography (CT)

Mass-spring models

Spline curves

Image features

\begin{abstract}
A B S T R A C T
We propose a computer-aided detection (CAD) system which can detect small-sized (from $3 \mathrm{~mm}$ ) pulmonary nodules in spiral CT scans. A pulmonary nodule is a small lesion in the lungs, round-shaped (parenchymal nodule) or worm-shaped (juxtapleural nodule). Both kinds of lesions have a radiodensity greater than lung parenchyma, thus appearing white on the images. Lung nodules might indicate a lung cancer and their early stage detection arguably improves the patient survival rate. CT is considered to be the most accurate imaging modality for nodule detection. However, the large amount of data per examination makes the full analysis difficult, leading to omission of nodules by the radiologist. We developed an advanced computerized method for the automatic detection of internal and juxtapleural nodules on low-dose and thin-slice lung CT scan. This method consists of an initial selection of nodule candidates list, the segmentation of each candidate nodule and the classification of the features computed for each segmented nodule candidate.The presented CAD system is aimed to reduce the number of omissions and to decrease the radiologist scan examination time. Our system locates with the same scheme both internal and juxtapleural nodules. For a correct volume segmentation of the lung parenchyma, the system uses a Region Growing (RG) algorithm and an opening process for including the juxtapleural nodules. The segmentation and the extraction of the suspected nodular lesions from CT images by a lung CAD system constitutes a hard task. In order to solve this key problem, we use a new Stable 3D Mass-Spring Model (MSM) combined with a spline curves reconstruction process. Our model represents concurrently the characteristic gray value range, the directed contour information as well as shape knowledge, which leads to a much more robust and efficient segmentation process. For distinguishing the real nodules among nodule candidates, an additional classification step is applied; furthermore, a neural network is applied to reduce the false positives (FPs) after a doublethreshold cut. The system performance was tested on a set of 84 scans made available by the Lung Image Database Consortium (LIDC) annotated by four expert radiologists. The detection rate of the system is $97 \%$ with $6.1 \mathrm{FPs} / \mathrm{CT}$. A reduction to $2.5 \mathrm{FPs} / \mathrm{CT}$ is achieved at $88 \%$ sensitivity. We presented a new 3D segmentation technique for lung nodules in CT datasets, using deformable MSMs. The result is a efficient segmentation process able to converge, identifying the shape of the generic ROI, after a few iterations. Our suitable results show that the use of the 3D AC model and the feature analysis based FPs reduction process constitutes an accurate approach to the segmentation and the classification of lung nodules.
\end{abstract}

(c) 2012 Elsevier Ltd. All rights reserved.

\section{Introduction}

Lung cancer is the leading cause of cancer deaths. The overall 5 -year survival rate is only $10-15 \%[1,2]$ and no significant improvement has occurred in the last 20 years [3]. Approximately $70 \%$ of lung cancers are diagnosed in too advanced stages thus making the treatments ineffective [4].

The nodule identification in screening CT is particularly difficult as low-dose CT images show a noisier appearance and an

\footnotetext{
* Corresponding author. Tel.: + 3909123899050.

E-mail address: donato.cascio@unipa.it (D. Cascio).
}

amount of image data as large as about 300 slices per scan. In order to support radiologists in the challenging task of interpreting screening lung CT scans, researchers have begun to explore recently computer-aided detection (CAD) methods for automatic identification of pathological objects in the images. The automatic segmentation of lung nodules is a difficult task due to the various anatomic situations in which the lung nodules may be found, leading to the four following main problematic situations:

- lung nodules often touch or infiltrate surrounding pulmonary structures (vessel, pleural), a process leading to the failure of all purely gray value-based segmentation techniques; 
- adjacent high-contrast structures often lead to high local gradient magnitudes, which may disturb model-based segmentation approaches relying on contour strength;

- the nodule shape is typically elliptical when well-circumscribed, but may vary significantly when attached to other pulmonary structures;

- the size of lung nodules varies significantly.

The different lung nodule detection CAD schemes can be classified according to various broad categories of approaches.

Intensity based schemes [5-8]: this type of approaches explores the intensity (voxel value) properties of the lung nodules in comparison with the surrounding background (tissue structure). Kostis et al. [9] adopted a morphological opening with a fixed size structure element aimed to separate small nodules from the attached vascularity, and an iterative dilation procedure for nodule borders reconstruction. Using multiple threshold levels combined with feature analysis (e.g., shape, size), Giger et al. [8] computed the likelihood of a suspected region representing an actual nodule. The primary advantage of this approach is its simplicity; the main drawback is the frequent difficulty to determine robust threshold levels for different CT examinations which implies a relatively low sensitivity and a high false positive detection rate. Brown et al. [10] use 3D segmentation involving attenuation thresholding, region growing and mathematical morphology to identify the regions of interest. The system labels these regions on the basis of a model of lung nodules and relevant intrathoracic anatomy. Gradient based schemes [11,12]: this generic type of approach consists of the analysis of the high gradient regions with the purpose to individualize the nodules contours. The approaches presented by Roy et al. [11] make use of the radial gradient index. For each CT section, high circularity regions are enhanced improving the contrast between the nodules and the non-pathological structures. Pu et al. [12] use a geometric model based algorithm. The main advantage of this approach is that using an efficient geometric model relying on the analysis of the signed distance field could minimize the effects of the large variability in lung nodule appearances.

Template based approaches $[13,14]$ are using statistical models for geometric or intensity-based features characterization of the lung nodules; these features are further used for searching matched object templates in the image space. In the method described by Fan et al. [13], a thresholding is followed by the analysis of the nodule's orientation and size, and the adaptation of a 3D template. The method suits the approximately ellipsoidalshaped small nodules, and requires interactive correction in case of the nodules with irregular shapes. However, for nodules attached to the chest wall, the ellipsoid shape is usually a bad approximation. Okada et al. [14] present an automated method to approximate the solid nodules as well as the ground glass opacities by ellipsoids with an anisotropic Gaussian fitting. The volume of the nodule is essentially given by the volume of the ellipsoid. While the approach is intriguing due to its applicability to non-solid nodules, the question of volumetric reproducibility for nodules of non-elliptical shape, especially in those cases in which the shape changes due to irregular nodule growth, is a potential drawback of this ellipsoid approximation approach.

Machine learning classifiers $[15,16]$ characterize nodules with features extracted directly from a reference database of known (verified) nodules and these features are used as training input by a learning machine. This approach generally requires a large data set for training and testing. Ge et al. [15] have proposed a linear discriminant classifier based on 3D features. This classifier turned out to have relatively good performance, which may still need further validation. Suzuki et al. [16] investigated a peculiar artificial neural network (ANN) pattern recognition technique called massive training artificial neural network (MTANN) approach, in the reduction of the false positives in the computerized detection of lung nodules in low dose computed tomography (CT) images. Within this technique, the matching process is often computationally extensive.

Despite significant progress in this area, up to now there are no optimal, widely accepted (and used) CAD schemes for lung nodule detection. The difficulty in achieving a highly successful scheme arises from the aspect diversity of the lung nodules (e.g., shape, size, intensity) and, perhaps at a similar importance level, from the varying appearances of normal tissue structures (e.g., vessels, pleura). Differences in CT image noise patterns can also affect CAD performance levels and robustness.

In this work we present a CAD system based on a 3D MassSpring Model that aims to search and detect efficiently lung nodules on low dose CT images. Our system uses the same scheme for the location of both internal and juxtapleural nodules. Our hypothesis is that the use of a Stable 3D Mass-Spring Model minimizes the impact of the large variability in lung nodule appearances and image based feature values on the performance of the scheme. Moreover, this model-based segmentation technique was thought to require lower run-times with respect to normal clinical analysis time of a CT scan (i.e. a few minutes). This paper provides a description of the approach and reports our test results.

\section{Data set}

Chest computer tomography (CT) is considered the best imaging modality for the detection of lung nodules. In the last few years, low-dose CT scans were shown to be effective for the analysis of the lung parenchyma [17] thus making possible the perspective of screening programs. A medical image data set is the starting point for important epidemiological and statistical studies. The development of a CAD system is intimately linked to collection of a data set of selected images [18]. The CAD system was also tested using the scans provided by the Lung Image Database Consortium (LIDC) consortium [19,20], a publicly available database of collected thoracic CT scans aimed to promote the development of CAD systems and the comparison of their performances. Although our CAD system has been more specifically designed for thin slices CT scans, the test on the LIDC database constitutes an useful comparison with other systems performances. Furthermore, since each nodule larger than $3 \mathrm{~mm}$ in the LIDC database is described in detail by a set of radiologic characteristics, the performance of the CAD on different types of nodules can be studied. The image collection contains only individual marks of nodules larger than $3 \mathrm{~mm}$ as resulting from LIDC and acquired up to 2009; a total of 84 CT scans containing 148 nodules. There are other LIDC cases in the NBIA that were restricted and those will be continuously added to the available data. The identification of the used data is as follows: from the LIDC case 1.3.6.1.4.1.9328.50.1.0024 up to the case 1.3.6.1.4.1.9328.50.1.0150 (note that the case numbers are not consecutive, but are in ascending order). The dataset is an international web-available resource, hosted by the National Biomedical Imaging Archive (NBIA, https://imaging.nci.nih.gov/ ncia/login.jsf), a searchable repository of in vivo images provided by the biomedical research community, industry, and academia. Note also that in this paper, a "case" refers to a CT scan of a single patient and the term "case" will be used synonymously with "CT scan". These scans were acquired using single or multislice CT scanners, with (peak) voltages between 120 and $140 \mathrm{kVp}$, X-ray tube current between 40 and $422 \mathrm{~mA}$, and slice thickness between 1.25 and $3 \mathrm{~mm}$. Each scan is provided along with its 

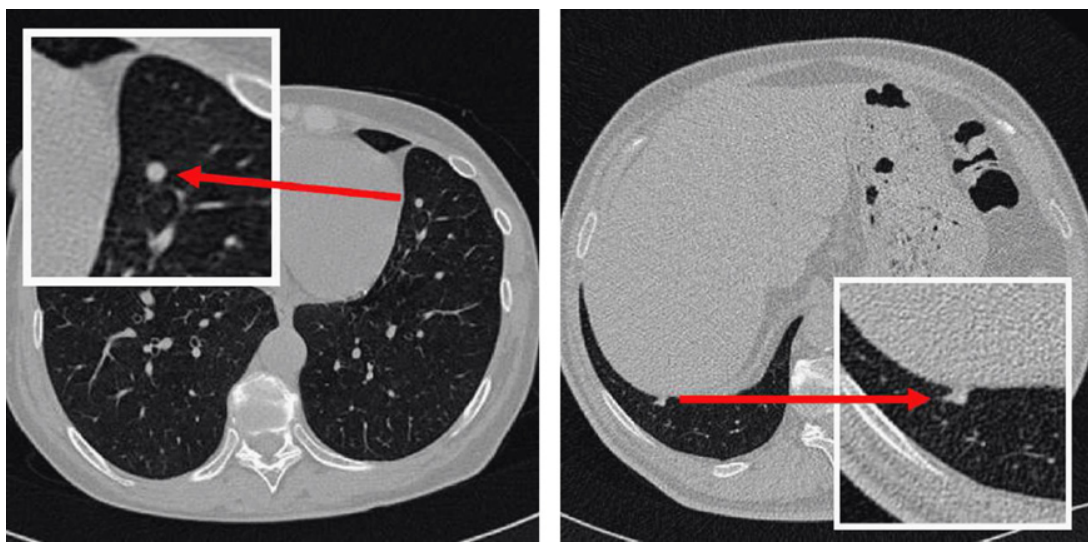

Fig. 1. Two examples of pulmonary nodules: internal nodule (left) and juxtapleural nodule (right).

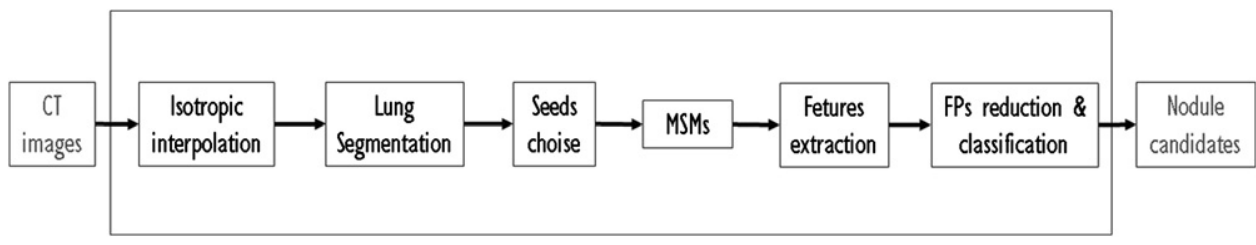

Fig. 2. CAD lung flow chart.

annotations by four experienced radiologists (each one from a different institution), who identified the location and radiological characteristics of all the nodules and other anomalies larger than $3 \mathrm{~mm}$ in diameter, as well as the location of nodules smaller than $3 \mathrm{~mm}$. Each radiologist performed first a blinded review of the scan, followed by an unblinded review with the information provided by the other radiologists available. No forced consensus was imposed. The annotations of all four radiologists in the unblinded review are available with the database. Only nodules larger than $3 \mathrm{~mm}$ were considered in this work. Fig. 1 shows two typical examples of pulmonary nodules: juxtapleural nodule (right) and internal nodule (left).

\section{Methods}

The strategy adopted in this paper for the automated detection of pulmonary nodules is based on the selection of nodule candidates by means of a stable 3D Mass-Spring Model (MSM) (introduced by Dornheim et al. [21,22]) and the reduction of the amount of false positives is based on a neural classifier. Our CAD scheme consists of six major steps:

1. isotropic interpolation;

2. lung parenchymal volume identification;

3. seed points choice;

4. detection and anatomical segmentation of regions of interest (ROIs) by the MSM;

5. features extraction for each ROI;

6. a neural network classification is performed in order to distinguish between true positive and false positive candidates after a double-threshold cut to reduce the false positives (FPs).

The flow chart of all these algorithms is displayed in Fig. 2. The main part of this work consists of the lung nodules segmentation based on a MSM process, that will be introduced in the following sections, coupled to a 3D reconstruction process carried out by spline curves. Details of the computerized scheme are described in the following.

\section{Isotropic interpolation}

Since the detection scheme relies on the volumetric analysis of CT images, we first convert all voxels into 3D Cartesiancoordinate grids with uniform 3D spatial resolution in order to reduce possible errors due to anisotropic representation of grids. Several interpolation techniques $[23,24]$ have been developed for this purpose. Among these, the linear interpolation is widely used for isotropic data reconstruction because of its computational simplicity. Considering that the spatial resolution along the axial direction (slice thickness) in CT examinations usually differs from the spatial resolution within each slice, we perform a linear interpolation along the axial direction. Regardless of the actual axial resolution, an interpolated slice is computed between all paired neighboring slices. Thus, at the end of the isotropic interpolation process the size of each voxel will be cubic.

\section{Lung parenchymal volume identification}

A crucial task for the nodule detection is the selection of the initial volume of interest (VOI). Lung segmentation is often used as a preprocessing step in CAD schemes. The lung must be segmented in order to reduce the computational time and the false positives number. The objective of the VOI segmentation algorithm implemented in this analysis is to allow a conservative identification either for the internal region of the lung parenchyma or for the region sufficiently close to the pleura surface. In both these regions we apply the nodule detection algorithm. Although threshold-based region filling strategies [25] can be used for a quite efficient extraction of the lung parenchyma, these strategies can also erroneously exclude important regions (i.e. juxtapleural nodules) when these regions are contiguous to the chest wall and have a similar density. Since juxtapleural nodule is attached to the pleura, a simple thresholding and filling process can never lead to separate identification of any juxtapleural object, it could at most identify a overall joint object far greater than just the pleura (with all consequent problems of classification. In this paper the input image, in DICOM (Digital Imaging and 
COmmunications in Medicine) standard format, is subjected to boundary segmentation using a two-step algorithm:

1. Including process: a 3D Region Growing (RG) method below a adaptive value threshold is used for identifying the parenchyma regions (right and left).

2. Morphological process: a dilation process is applied for including the internal structures with high intensity value (i.e. nodules, vessels, etc.) and of the pleural layer adjacent to the internal lung volume (the region containing the juxtapleural nodules).

\subsection{Inclusion process}

The internal lung volume consists of air and the bronchial tree, typically displayed in a CT slice as low intensity voxels surrounded by high intensity voxels corresponding to the pleura (see Fig. 3, obtained from one of the analyzed CTs). This suggests the segmentation of the internal lung volume by the means of a 3D RG algorithm which has the ability to create regions with similar characters. The basic approach consists of starting with a set of "seed" points from which the regions are subsequently grown by appending to each seed those neighboring points that have properties similar to the seed (inclusion rule); the process runs until there are no more points satisfying the predefined criteria. The choice of the inclusion rule with the optimal threshold and the selection of a proper seed point are of great relevance for a better performance of the algorithm. We choose as inclusion rule the Simple Bottom Threshold (SBT): if the intensity I of a generic voxel is lower than a threshold value, the voxel is included in the growing region. This threshold value is automatically selected through the method adopted by Ridler et al. [26] as result of an iterative process. It is based on the CT voxels gray value distribution which typically displays two quite distinct parts (Fig. 3): one containing air, lung parenchyma, trachea, and bronchial tree, the other one containing vascular tree, bones, muscles, and fat. The optimal threshold, is set at the plateau between these two regions.

\section{CT Gray Level Distribuition}

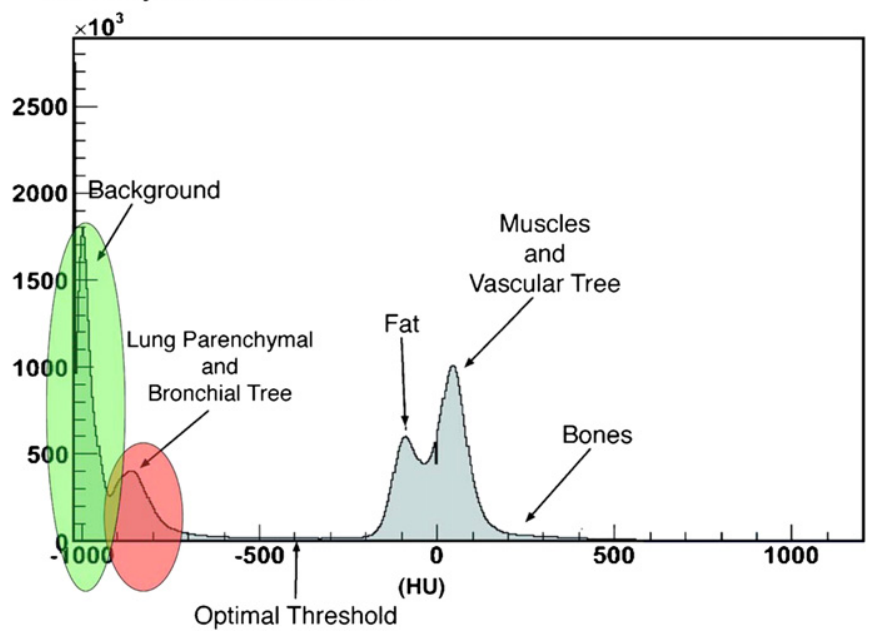

Fig. 3. Gray-level distribution of the voxels of one of the analyzed CT. Similar distributions can be obtained from the other CTs. The region segmented by the RG algorithm refers to the lung parenchymal and the bronchial tree. The voxels corresponding to the background, though having an intensity lower than the threshold, are not included in the grown region because they are not geometrically connected with the seed point. (For interpretation of the references to color in this figure caption, the reader is referred to the web version of this article.)
The RG algorithm uses two seed points. These seeds points have been selected according to the following scheme:

1. set the start point on the central voxel $\left(P_{0}\right)$ of central slice of the scan;

2. shift to the left of a voxel;

3. if the inclusion rule for such voxel is satisfied, then start the RG, else go to the step 2;

4. if, at the end of the recursive process of RG, the volume (in voxel) selected by the RG process has the same order of magnitude with the expected lung volume, the trial phase ends else go to the step 2. An information on the lung volume's order of magnitude is derived from the histogram of the CTs gray tones, by estimating the area under the second peak from the left (red region in Fig. 3). This part of the distribution, in fact, refers precisely to the voxels belonging to the lung parenchymal and bronchial tree.

For the selection of the right pulmonary volume, the steps 1-4 are repeated with a right shift (instead of the left shift) at the step 2. Fig. 5 displays a binary example of the inclusion process result.

\subsection{Morphological process}

At this stage, the internal nodules, vessels and airway walls are not included in the segmented lung. A dilation morphological operation is applied for segmenting the lung, filling the holes and including the pleural layer, where juxtapleural nodules are to be found. This is a 2D method applied to each single slice of the CT scan.

A collateral effect of the expansion phase may be the merging of the two volumes (right and left lung), as depicted in Fig. 6, but this effect does not affect the following stages of the analysis because, as previously stressed, the purpose of lung volume parenchymal identification is the reduction of the investigation volume (involved in nodule search). At the end of the segmentation process the trachea is also included since its density is similar to the lung parenchyma. A more accurate processing aimed to exclude the merging of the two volumes would involve more complex algorithms and significantly higher running times. An example of volume reconstruction is shown in Fig. 4.

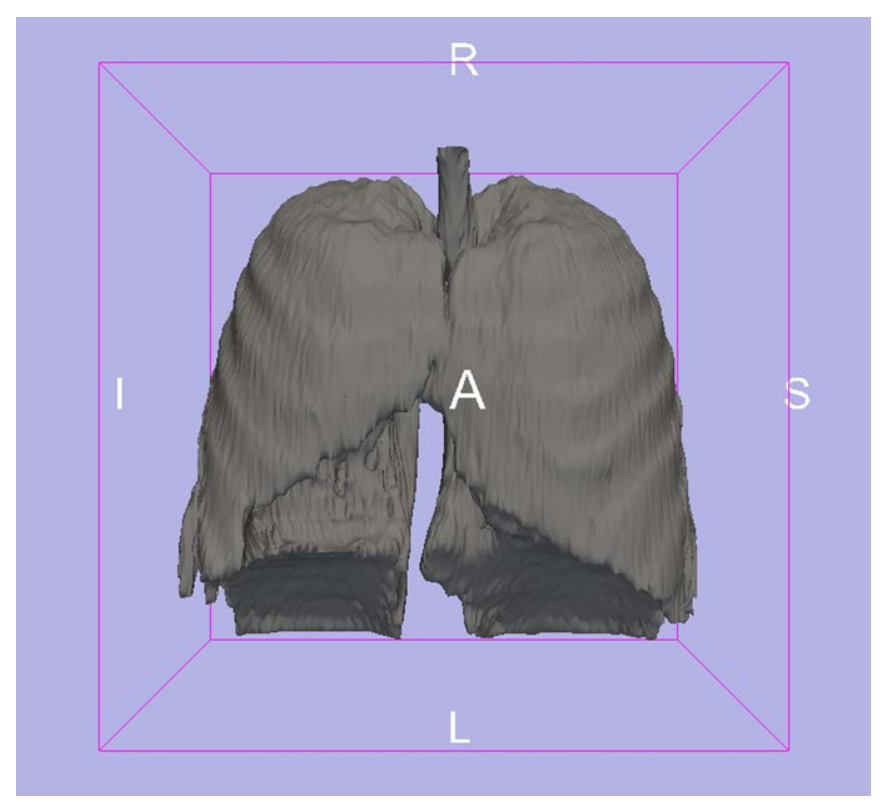

Fig. 4. Volume of interest 


\section{Seed points choice}

Since the nodules have a greater intensity with respect to the pulmonary parenchyma or to the air contained in the lungs, they can be found by looking for the local intensity maxima inside the pulmonary volume included after the dilation operation [27]. Therefore, a voxel level substraction is performed, involving the following matrices:

$A(x, y, z)=[D(x, y, z)-R(x, y, z)] \cdot I(x, y, z)$

where $R(x, y, z)$ is the binary matrix of the $3 \mathrm{D}$ image resulting from the RG process (Fig. 5), $D(x, y, z)$ is the binary matrix obtained at the end of the dilation process (Fig. 6$)$, and $I(x, y, z)$ is the input image. The result is a third 3D matrix $A(x, y, z)$ which will be used for the identification of the structures inside the pulmonary volume through a subsequent search of intensity maxima. Fig. 7 illustrates the application of the Eq. (1) to a slice.

A peak detector algorithm is applied to the matrix $A(x, y, z)$ to detect the local maxima and to generate the seed points list for the next step of nodules research. In a normal data scan, the average number of local maximum points found is about 2000; this is due to the similar intensity values for the blood vessels and the nodules.

\section{The stable 3D mass-spring models}

\subsection{Introduction to mass-spring models}

The main task in a nodule detection process is the segmentation of regions of interest (ROIs). There are a very large number of

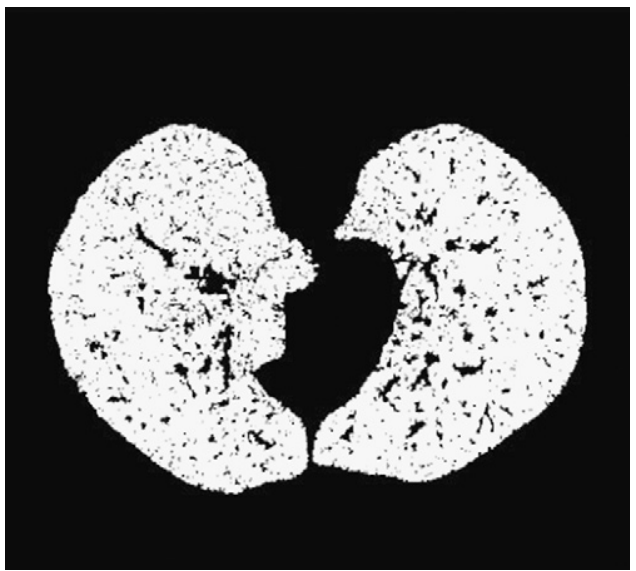

Fig. 5. Example of an including matrix resulted from region growing (a slice).

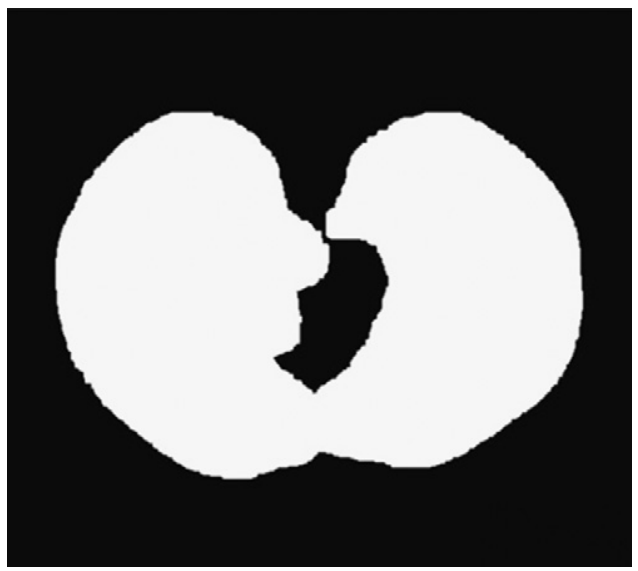

Fig. 6. The matrix after the morphological process (a slice).

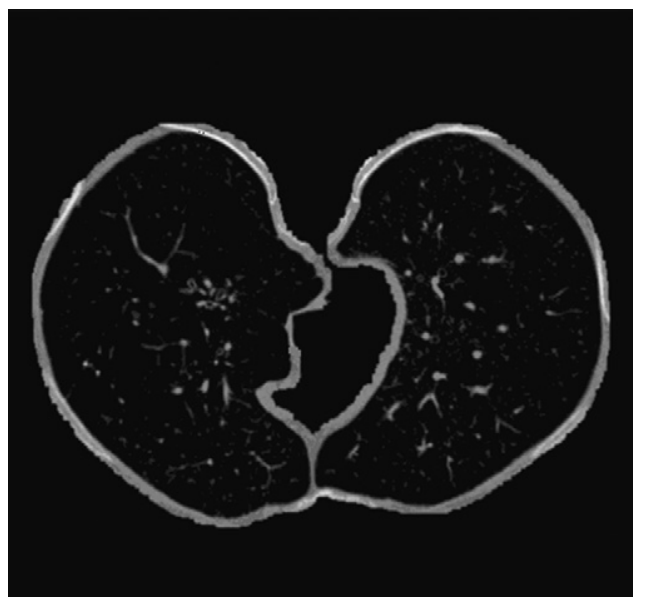

Fig. 7. Subtraction matrix (a slice): inclusive pleura are visible.

segmentation algorithms in the literature. A characteristic of the segmentation process, most of the involved algorithms are specific to the application, thus bearing little or no significance for most other applications.

Nodules are frequently attached to other structures, including the local pulmonary vessels and the pleural surface adjoining the thoracic wall. The geometry of such attachments should be taken into account in order to successfully segment each nodule type.

Mass-spring models (MSMs) are physically based models of usual application in soft-tissue simulation. These models consist in a network of mass points interconnected by elastic springs modeling their interaction, aimed to describe the shape of an object. Stable MSMs extend conventional MSMs not only by incorporating the springs rest lengths but also their rest directions. Whenever a spring deviates from its rest direction, a torque appears tending to restore its original orientation. We chose to use a stable MSM for the lung nodules segmentation for the following four major reasons:

1. the use of a Stable 3D mass-spring model minimizes the impact of the variability in lung nodule appearances (internal nodules and juxtapleural nodules) and of the image-based feature values, on the scheme performance;

2. the volumetric sampling of the dataset by mass points allows the joint concurrent representation in the model of the contour and the gray value information;

3. MSMs permit fast and flexible model creation;

4. the use of spring forces and torques distinguishes between model's size and shape, a feature allowing to represent the lung nodules shape while leaving the size of the target lung nodules flexible.

From a specific medical imaging perspective, a very important advantage of these models is their capability to accommodate the often significant variability over time of the biological structures.

MSMs can also be applied as deformable models for segmentation. In this case, no simulation of a real physical situation is required. This process is comparable to the adaptation of an active contour model and aims to find an equilibrium between the internal forces, describing the model shape, and external forces, describing the image information. In the physics-based modeling paradigm, the shape corresponds to applied (external) forces on the deformable model and consequently, the model moves towards the shape, while internal forces keep the model smooth during deformation. Deformable models gained popularity after they were proposed to be used in computer vision [28] and computer graphics [29] by Terzopoulos and others in 1988. 
Mathematically, a deformable model moves according to its dynamic equations and seeks the minimum of a given energy function. The deformation of a typical 2D deformable model can be characterized by the following dynamic equation:

$\mu(s) \frac{\partial^{2} X(s, t)}{\partial t^{2}}+v(s) \frac{\partial X(s, t)}{\partial t}=\mathbf{F}_{i n t}+\mathbf{F}_{e x t}$

where $X(s, t)=(x(s, t), y(s, t))$ is a parametric representation of the position of the model at a given time $t, s$ is the parameter characterizing the parametric curve $(s \in[0,1]), \mu(s)$ and $v(s)$ are parameters representing the mass density and the damping density of the model, respectively.

The most commonly used internal forces are

$\mathbf{F}_{i n t}=\frac{\partial}{\partial s}\left(\varphi(s) \frac{\partial X(s, t)}{\partial t}\right)-\frac{\partial^{2}}{\partial s^{2}}\left(\phi(s) \frac{\partial^{2} X(s, t)}{\partial t^{2}}\right)$

which represent internal stretching and bending forces: $\varphi(s)$ controls the "tension" of the contour while $\phi(s)$ controls its "rigidity". The most commonly used external forces are computed as the gradient of an edge map.

The goal of the energy minimization formulation approach used here is to find a parametric model that minimizes the weighted sum of internal energy and external energy. The internal energies impose constraints on the contour itself, while external energies push the contour toward salient image features such as lines and edges. Our choice, as discussed in detail in the following paragraphs, was to evolve the MSM model using the method of energy minimization.

\subsection{Design and initialization of the 3D mass-spring models}

In this work we developed an appropriate system of forces (and thus, an appropriate energy functional) able to provide solutions corresponding to nodule contours. We represent the contour by a set of $N$ mass points model vertices. We took a sphere mesh of masses connected by springs (Fig. 8) as initial 3D MS Model. The size of the sphere mesh was chosen in such a way to contain nodules with sizes in the range of some centimeters. The basic idea of the model is that, starting from an "enlarged" position, the mass-spring mesh grips the potential nodule. Each of the seed points found by the procedure previously described in the section "Seed Points Choice" is taken as center of a mesh massspring.

Labeling with $N$ the number of mass points that make up the active model and with $n$ the number of points of the model on a single slice, the relation $N=n \cdot s$ holds, where $s$ is the number of

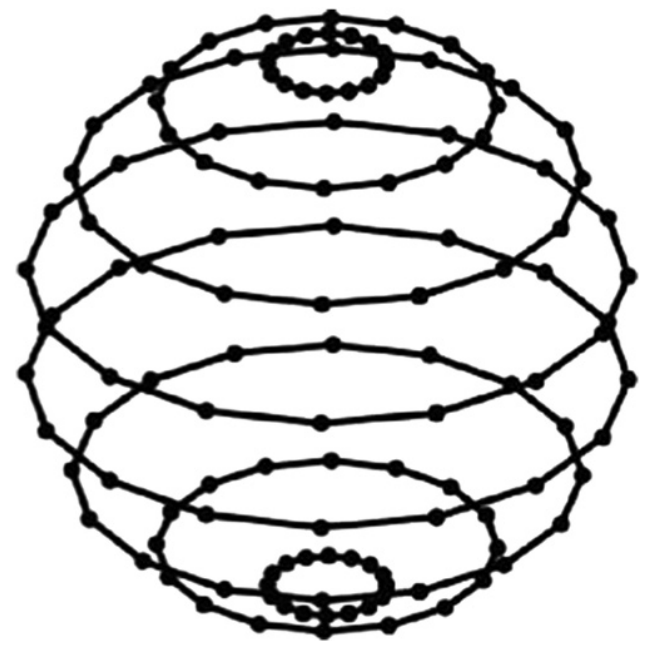

Fig. 8. The model is initialized by placing the masses on the surface of a sphere.

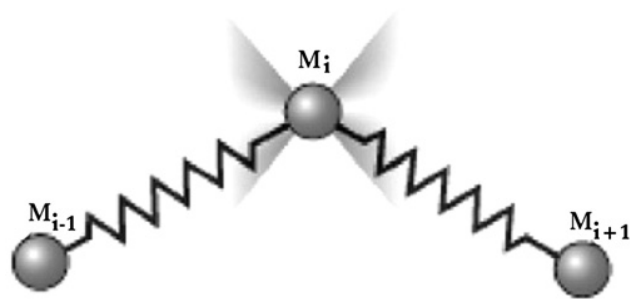

Fig. 9. The broad mass $M_{i}$ of the model is linked to two other masses of the same slice through as many springs.

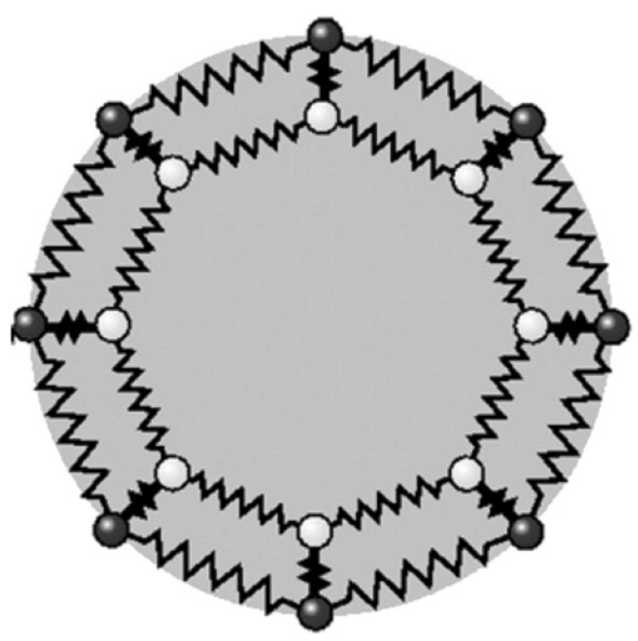

Fig. 10. Springs and masses from two slices during the evolution model.

slices involved in the search for a single nodule. Each mass $M(i)$ $(i=1, \ldots, N)$ is connected through two springs with two other masses, $M(i-1)$ and $M(i+1)$, belonging to the same slice (Fig. 9) and with two masses, $M(i-n)$ and $M(i+n)$, belonging to the previous (Fig. 10) and to the following slice respectively:

$E_{\text {functional }}^{\text {tot }}=\sum_{i=1}^{N}\left[E_{\text {internal }}(i)+E_{\text {external }}(i)\right]$

Each point of the model contributes with both internal and external energies to the energy functional.

\subsection{Internal energy}

The energy $E_{\text {internal }}(i)$ of a generic vertex $i$ of the mass-spring model is computed by summing up the following contributions:

- Elastic energy: this energy makes the model contract in order to adapt itself to the shape of the object to be segmented. The generic vertex labeled $i$ is virtually connected to its four first-order neighbors by four springs (two in the $x y$-plane and two parallel to the $z$ axis), which contribute with an elastic energy $E_{\text {elas }}(i)$

$E_{\text {elas }}(i)=\alpha\left[E_{s p r, i-1}(i)+E_{s p r, i+1}(i)+E_{s p r, i-n}(i)+E_{s p r, i+n}(i)\right]$

where $E_{s p r, j}(i)$ is the elastic energy of the spring connecting the two masses $i$ and $j$

$E_{s p r, j}(i)=\frac{1}{2} k \Delta x^{2}$

where $\Delta x$ is the distance between the two masses and $k$, the spring constant, is a parameter.

- Bending energy: in addition to contraction, the model must adapt itself to the different curvatures that the object may exhibit. Therefore, the effect of the bending term is to force the mass points of the model by penalizing those positions of the 
masses leading to high curvatures. The bending energy associated with the $i$ th vertex is defined as follows:

$E_{\text {bend }}(i)=\beta\left\|P_{i-1}-2 P_{i}+P_{i+1}\right\|^{2}$

where $P_{i}$ indicates the position of the $i$ th vertex. In order to prevent the twisting of the active contour during model's evolution, a check is carried out and the vertices configurations corresponding to angles excessively small or large are cut off.

- Attraction energy: the geometric center of the positions of the vertices belonging to a same slice is used as reference point for the calculation of the distance to the vertices average $(\bar{d})$ and standard deviation $\left(\sigma_{d}\right)$. If the $i$ th vertex is placed at a distance $d_{i}$ from the center such as

$d_{i}>\bar{d}+\sigma_{d}$

then to the vertex $i$ is associated with an attraction energy $E_{\text {attr }}(i)$ given by

$E_{\text {attr }}(i)=\gamma \frac{d_{i}}{\bar{d}}$

Therefore, farer is a vertex from the center with respect to the average distance, greater is the contribution of additional energy. This contribution favors more regular trends for the points on the model surfaces, making the latter almost spherical as those of the nodules. This term also prevents a vertex from remaining stationary in a position of local minimum (e.g. caused by noise in the $\mathrm{CT}$ ) far from other vertices.

\subsection{External energy}

The internal energy evaluated on the contour points regulates the contraction and bending, but does not provide any kind of useful "pulling" force on the desired segmented object volume. In other words, we need to combine the energy contributions in such a way that the model's evolution converges towards our target surface, that is on the boundary points separating an object (in our case a nodule) from the rest of the image. The external energy has therefore the role to attract the model on the object edges. The energy $E_{\text {external }}(i)$ of a generic vertex $i$ of the mass-spring model is computed as the sum of the following contributions:

- Gradient energy: this energy contribution makes the points of the model evolve towards locations with high gradients; this behavior corresponds to the typically high gradients exhibited within an image by the contour points of a generic nodule. A little energy amount will be associated to the generic ith vertex if located on an edge point, given by the following equation:

$E_{\text {grad }}(i)=-\delta\left\|\nabla I\left(P_{i}\right)\right\|$

where $I\left(P_{i}\right)$ is the intensity at the position $P_{i}$ of the $i$-th vertex.

- Potential energy: to each point of the model is associated a potential energy $E_{p o t}$ proportional to the value of the image intensity at that position: "brighter" is the point, more energy is associated with it. Thus, if a point moves from a position with a low intensity value (such as the outer edge of a nodule) to another position with higher intensity (the pleura or the region's internal nodule itself), it must "pay" an additional contribution of energy

$E_{\text {pot }}(i)=I\left(P_{i}\right)$

\subsection{Energy functional}

The energy $E_{\text {functional }}(i)$ of a generic vertex $i$ of the mass-spring model is computed as the sum of the following terms:

$E_{\text {functional }}(i)=\alpha E_{\text {elas }}(i)+\beta E_{\text {bend }}(i)+\gamma E_{\text {attr }}(i)+\delta E_{\text {grad }}(i)+\epsilon E_{\text {pot }}(i)$ where $\alpha, \beta, \gamma, \delta$ and $\epsilon$ are dimensional parameters empirically deduced. In order to determine the values of these parameters we introduced various sized artificial objects in the images: spheres (representing the internal nodules) and hemispheres (representing the internal juxtapleural nodules). The objects have been injected with different sizes (the size of simulated nodules has been constantly kept inside the interesting range [3-50] $\mathrm{mm}$ ), and for each object has been evaluated the corresponding reconstructions in terms of differences between expected values and obtained values for two extracted features: the sphericity (must be 1 for the sphere and 0.84 for the hemisphere) and the radius. A further statistical analysis performed on 50 spheres and 50 simulated hemispheres led us to the choice of these parameters. The approach of inserting a simulated object inside a "real" context has been chosen for process optimization purposes in the presence of noise (the background).

The initial vertices of the mass-spring model, initialized as sphere mesh of spring-connected masses, are subsequently adjusted by a step-by-step process until a minimum of the total energy functional is reached

$E_{\text {functional }}^{\min }=\min \left(\sum_{i} E_{\text {functional }}(i)\right)$

\subsection{Evolution of the model}

We designed a convergent procedure for the evolution of the mass-spring model from an initial state in which the vertices are laid out on a large-sized box (this box can contain ROI sizes up to $50 \mathrm{~mm}$ ). From this type of initial condition the model is willfully supposed to converge towards the generic ROI contours by a contraction. The procedure we developed has the following properties:

- low computational complexity;

- easiness of implementation;

- excellent performance if the initialization of the model is not very far from the target (as is the case in our application).

The steps of the algorithm are the following:

1. the value of the energy functional $E_{\text {functional }}(i)$ is initialized with the energy value calculated at the $i$ th vertex $(i=1, \ldots, N)$ of the initial sphere mesh;

2. For each vertex, the algorithm evaluates the energy functional for the vertex itself and for its eight (first-order) neighbors belonging to the same slice (the generic vertex is allowed to move only within its own slice). The new vertex location is subsequently chosen as the lesser energy vertex among these nine possibilities. The iteration process finishes when a full cycle energy analysis of all the $N$ vertices is completed;

3. if a vertex remains stationary for two successive iteration steps, it will be artificially shifted by an applied "jolt"; the procedure corresponds to the broadening of the neighborhood where the energy functional gets estimated to successive orders of neighbors. For the energy analysis, these new locations are chosen along the direction towards the geometric center of the model;

4. if the energy functional of $N$ points resulting from Eq. (4) is less than the energy functional estimated at the previous iteration step, then the procedure returns to the step item (2), otherwise the minimization process ends.

For the segmentation of a generic object, the algorithm requires an average of 11 iteration steps corresponding to a computational 

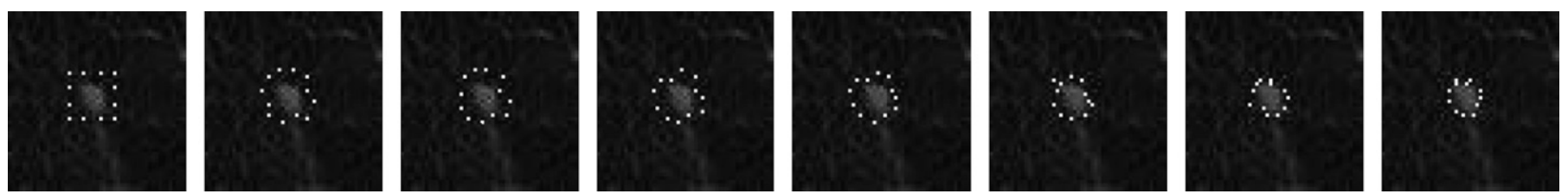

Fig. 11. Sample of MSMs analysis on a internal nodule: visualization of the evolution on a central slice with cubic initialization.
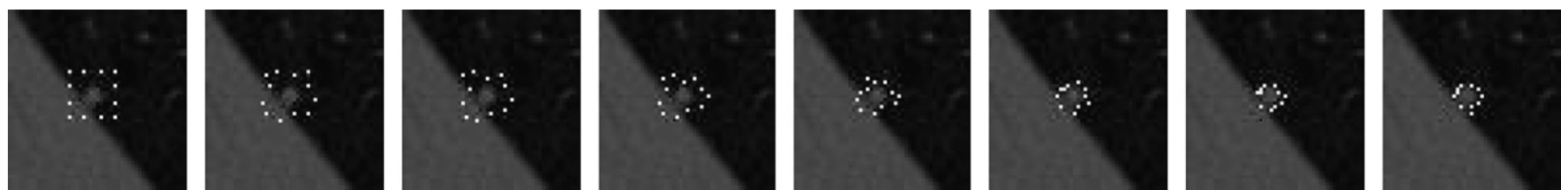

Fig. 12. Sample of MSMs analysis on a juxtapleural nodule: visualization of the evolution on a central slice with cubic initialization.
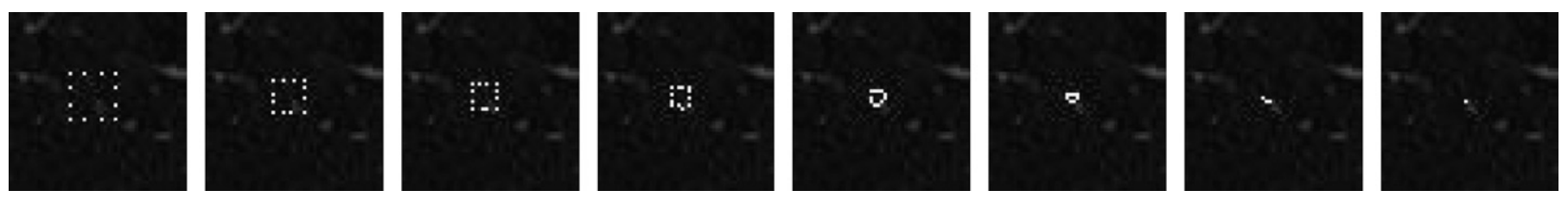

Fig. 13. Sample of MSMs analysis on a noise area: visualization of the evolution on a central slice with cubic initialization.

time of about $0.04 \mathrm{~s}$. The segmentation process of a standard CT image completes in about $1.5 \mathrm{~min}$; on a Linux-based PC system characterized by an AMD Athlon 4200, double core processor and $4 \mathrm{~GB}$ of physical memory. Obviously, computational times for evolving the MSM on a certain object depend on the number of performed iterations. Since the model evolves through the shrinking of an initial extended sphere up to the identification of object's boundary, there is also a statistical dependence of the computational time on the dimensions of the analyzed object: smaller the object to be segmented, larger the average number of performed iterations.

Figs. 11 and 12 illustrate the typical convergence sequence of the model on internal and juxtapleural nodules. Fig. 13 illustrates the "collapse" of the model in an area deprived of nodules (the seed is a noise point).

\subsection{The surface reconstruction with spline curves}

The output of the previously described mass-springs model consists of a 3D array of points $(x, y, z)$ containing the ROI as a "net". The next elaboration phase is the contour identification by a fit of these points, a procedure which leads to a clean separation between the generic ROI and the rest of the image; in fact, greater is the accuracy with which the generic ROI comes "drawn out" from the image, greater will be the precision with which the ROI will be analytically represented. This surface representation/ reconstruction problem can be stated in mathematical terms as follows:

given $N$ distinct points $\left(x_{i}, y_{i}, z_{i}\right)(i=1, \ldots, N)$ lying on a surface $M$, find a reasonable approximating surface $M^{+}$for $M$.

We have tried an original approach in solving the reconstruction problem and we opted out for using the 2D splines as described in the three following steps:

1. interpolation of the model contour points on a slice (the xyplane); the contour points are interpolated on a same slice with the imposed condition of identity of the first and the last point of the curve;

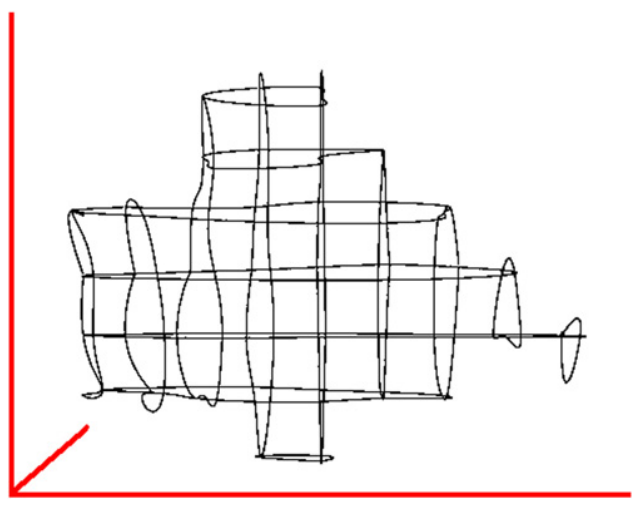

Fig. 14. An example of splines reconstruction.

2. identification of the checkpoints for tracing the spline in the $y z$ plane; in order to get a valid 3D reconstruction, the checkpoints obtained by intersecting the splines resulted at the previous step and the $y z$-planes are required for the final interpolation;

3. interpolation of the checkpoints for spline construction (yz-plane); the checkpoints are subsequently interpolated in order to get some splines along the $y z$ plane (the planes not containing points are obviously rejected). All the splines issued at this last step define our best 3D representation of the segmented ROI.

An example of reconstruction with splines is shown in Fig. 14, while Fig. 15 displays an example of a reconstructed nodule.

\section{Features extraction}

Once the 3D shape and volume of the nodule are obtained, all seven commonly used features [30-34] based on shape, size, intensity can be extracted. In this study, along with the three geometrical features (surface, volume and sphericity), we also use 


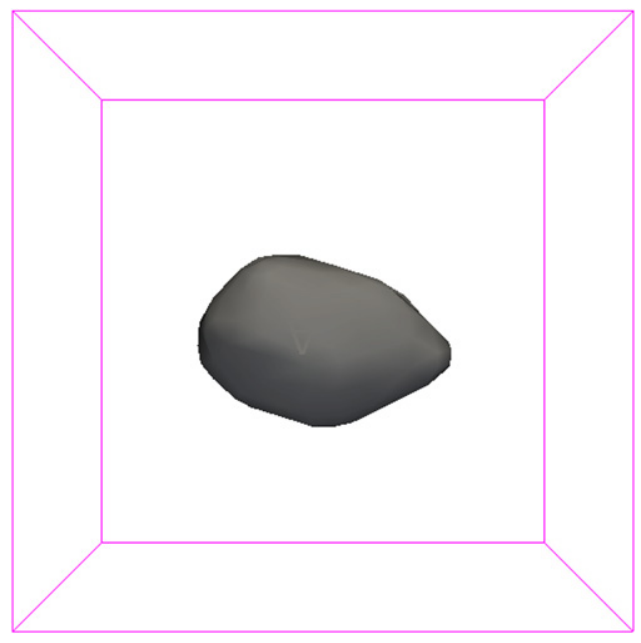

Fig. 15. The relative 3D nodule reconstruction.

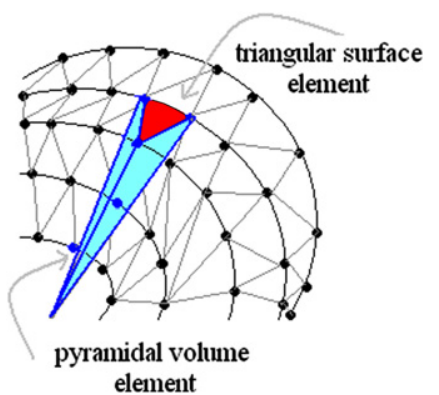

Fig. 16. Calculation of the surface and volume of the ROI: the surface is split into triangles, the volume is split into triangular pyramids.

four intensity distribution features (mean, standard deviation, skewness and kurtosis). As known from the literature, these features are defined as follows:

1. surface area: the area of a generic ROI is computed as the sum of the areas of the triangular elements derived from the points of the previous reconstruction phase (Fig. 16);

2. volume: the volume is computed as the sum of the pyramidal volume elements with the bases on the triangular surface elements having the ROI center as vertex (Fig. 16);

3. sphericity: this parameter measures the degree of similarity between the surface of an object and a sphere. In mathematical terms, the sphericity is given by the ratio between the surface area of a sphere (with the same volume as the given object) and the surface area of the object

$S=\frac{\pi^{1 / 3}(6 V)^{2 / 3}}{A}$

where $V$ and $A$ denote the volume and the surface area of the nodule candidate. The sphericity $S$ of a purely spherical shape is 1 while for a hemispherical shape one has $S=0.84$. Therefore we can measure how different is a nodule shape from a hemispherical form;

4. mean of the nodule intensity: the mean value of the gray level of the voxels inside the nodule candidate volume;

5. standard deviation of the nodule intensity: the standard deviation measures the fluctuation of the gray level of the voxels of the nodule candidate;

6. skewness of the nodule intensity: also known as distribution's third (central) moment, the skewness is a statistical parameter characterizing the distribution just as the mean value (i.e. the first central moment) and the standard deviation (i.e. the second central moment). The skewness is a measure of the lopsidedness of the distribution; in particular the skewness of a symmetric distribution is zero. In our case, the computation of the skewness has been performed on the gray level distribution of a generic ROI and provides an useful information about it, therefore it can be treated as a feature. This parameter is given by

Skewness $=\frac{\sum_{i=1}^{N}(g(i, j, k)-\mu)^{3}}{N \sigma^{3}}$

where $N$ is the number of voxels of the ROI, $g(i, j, k)$ is the gray level at location $(i, j, k), \mu$ and $\sigma$ are the average intensity and standard deviation inside the segmented nodule candidate, respectively. If the skewness is negative the data are spread out more to the left of the mean than to the right; if it is positive, the data are spread out more to the right;

7. kurtosis of the nodule intensity: a measure of the histogram "peakedness". It is defined as follows:

Kurtosis $=\frac{\sum_{i=1}^{N}(g(i, j, k)-\mu)^{4}}{N \sigma^{4}}$

We can investigate how different is the intensity distribution of the nodule from a normal distribution. The kurtosis value of the normal distribution is 3 .

\section{FPs reduction and classification}

In the present work, the previously defined features have been used in two different algorithms, both aimed to reduce the occurrence of false positives:

- identifying a simple double-threshold cut: the number of ROIs obtained by the MSM selection procedure on LIDC dataset is large: 33 642. Almost all FP findings refer to candidate ROIs with too many or too few voxels, and can be easily ruled out by a simple double-threshold cut on the volume V. The diameters of the structures of interest to radiologists range between $3 \mathrm{~mm}$ and $50 \mathrm{~mm}$. Hence, we exploit this prior information for ruling out all the findings with sizes ranging out of this interval and eliminating thus the false positives found by the nodule hunter. It should be emphasized that the threshold values arise from the diagnosing settings and do not rely on the structures found by the nodule hunter. For the other derived features, the double-cut on the findings was obtained identifying, for each features $(f(i))$, the pathology range $\left[f_{\min }(i), f_{\max }(i)\right]$; the cut-off is performed outside the pathology range. While systematically used [7], this method exhibits a dependence of the detected cut-off values on the actual pathologies sample used by it. Thus, for cut-off values computed on the basis of a small sized pathologies sample, the pathology ranges could be underestimated; hence, on a new data sample given as CAD input, there could be some sensitivity loss (although minimal) due to this processing phase. In our specific case, disposing of a rather good data sample (148 nodules) makes us pretty confident about the reliability of the results presented in this paper. However, as reported in the "results" section, for acquiring even more confidence with system's capabilities and for estimating the range of the bias introduced by this procedure, the system classifier has been trained without applying the double-threshold cut, maintaining still the volumetric cut because it identifies the investigated pathology. As result of this double-threshold cut the efficiency is preserved(100\%), while FPs are reduced to 2335 corresponding to $28 \mathrm{FPs} / \mathrm{CT}$. 
- classifying the input parameters with neural network application: a further reduction of the FPs can be obtained by means of a classification step. This phase of classification has been implemented with a three layered feed forward neural network; the neural network underwent a supervised learning training based on the algorithm of back-propagation with momentum, and its structure is the following:

o first layer with seven input neurons;

o second layer with three hidden neurons;

$\checkmark$ third layer with one output neuron.

The leave-one-out nodule cross validation [7,35] technique is used to exploit the highest possible number of TPs during the training phase, without invalidate the results. The name of this algorithm reflects its computational strategy: at each step, one of the TP patterns is successively left out and used for the validation of the network while the other ones are used for its training. As far as the FPs are concerned, a subset of proper size must be selected for a suitable training of the network. To decrease the number of subsets (otherwise it would get 148 subsets), the number of TP patterns successively left out, in this work, is four instead of one. After an extensive investigation of various trialand-error approaches, we have chosen to use all the 148 TPs and 888 FPs for the training phase, proceeding as follows:

- the 888 FPs are randomly extracted from the 2335 FPs, thus ensuring the representativeness among all FPs of the FP pattern set used for the network training;

- the patterns (148 TPs + 888 FPs) are randomly divided into 37 sets, each one containing 4 TP and 24 FPs;

- 36 out of the 37 sets are put together apart as training input for the network, while the last one is used for testing: in this way a neural output is assigned to each pattern of the test set;

- a cyclical permutation of the sets is carried out: for each permutation the network is trained on 36 input sets and validated on the remaining set; a neural output is thus assigned to all the patterns ( 148 TPs +888 FPs);

- the remaining 1447 FPs having not been selected for the cross validation are divided into 37 subsets; each subset is classified by one of the 37 networks.

At the end of this routine, a neural output is assigned to all 148 TPs and 2335 FPs. After the 37 trainings we have 37.4 TPs patterns and 37.24 FPs patterns for the test set, independent because they are systematically not used in the training phase. All these data contribute with good statistics to the FROC curve in Fig. 17.

\section{Results}

Fig. 17 shows the FROC curve which describes the nodule detection overall performance of the CAD system, a parameter summing up the contributions of the effectiveness of the lung volume segmentation, of the nodule candidate searching and of the FP reduction mechanism. As one can note from Fig. 17, the use of the neural network reduces the FPs/CT ratio from 28 to 6.1, with a correlated sensitivity reduction from $100 \%$ (its value after the double-threshold cut) to $97 \%$. A further reduction to $2.5 \mathrm{FPs} /$ CT can be achieved with a lower detection rate (88\%).

A direct comparison between our results and the performance of other CAD systems is unfeasible if:

1. the databases used by other CAD systems differ in the number of $\mathrm{CT}$ scans, the number of slices per $\mathrm{CT}$ and the dose of the images (in some cases this information is missing);

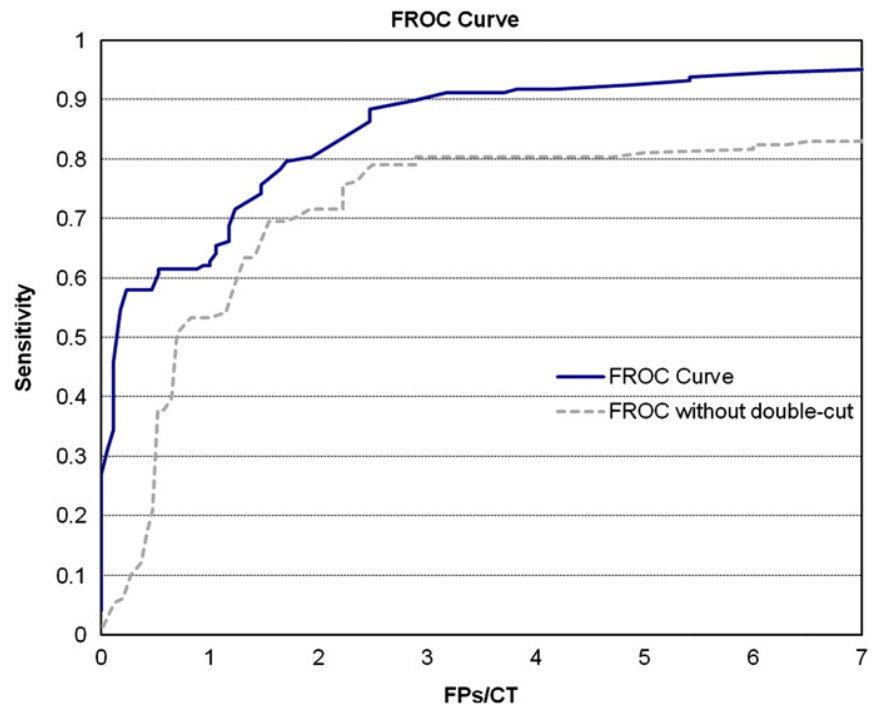

Fig. 17. Overall CAD FROC curve reporting the sensitivity (sensitivity is the fraction of positive nodule candidates correctly classified), evaluated with respect to the radiologist's diagnosis, against the FPs/CT. The figure presents, as dashed line, the FROC curve of the system's performance without using the doublethreshold cut.

Table 1

Review of the results reported by other CADs: the asterisk indicates those works that have been validated on the LIDC dataset of 84 CTs scan

\begin{tabular}{llrrrrrr}
\hline CAD & & Scans & Slices/CT & Nodules & Efficiency & FPs/slice & FPs/CT \\
\hline Bellotti & {$[7]$} & 15 & $\sim 314$ & 26 & $80 \%$ & - & 2.47 \\
Brown & {$[10]$} & 8 & 40 & 22 & $86.4 \%$ & - & 2.64 \\
Roy & {$[11]$} & 38 & $\sim 51$ & 82 & $70 \%$ & 0.28 & - \\
Pu & {$[12]$} & 52 & - & 184 & $81.5 \%$ & - & 6.5 \\
Suzuki & {$[16]$} & 63 & $\sim 28$ & 71 & $80.3 \%$ & 0.18 & 4.8 \\
Golosio* & {$[36]$} & 84 & $\sim 310$ & 148 & $71 \%$ & - & 4.0 \\
Messay* & {$[37]$} & 84 & $\sim 310$ & 143 & $82.7 \%$ & - & 3.0 \\
Our CAD* & & 84 & $\sim 310$ & 148 & $97 \%$ & - & 6.1 \\
& & & & & $88 \%$ & - & 2.5 \\
\hline
\end{tabular}

2. the starting points of the processing systems are different: our CAD processes the whole CT and automatically extracts the slices to be analyzed;

3. the results are expressed in different ways: in particular, FPs are given per slice or per CT (it should be hinted out that in general the FPs/CT ratio differs from the product of the FPs/ slice ratio with the number of slices/CT);

4. some CAD systems focus their results analysis on the nodules dimension, thus providing detection rates at different nodule sizes.

Notwithstanding this, we believe it is still important to attempt a relative comparison. We have identified published results $[36,37]$ that have used the same LIDC dataset scans to validate detection systems. A summary of the results achieved by other CAD systems is given in Table 1 . In this table, each row represents a published benchmark followed by the scans number, average value of slices for scan, nodules number and the optimized operating point reported by the according system. Based on the very comparison with the two systems operating on the same image CTs, one can infer that the performance of our proposed system is at least on a same level with the results displayed in the mentioned papers. Concerning the use of the 
double cut-off, the key figure is the comparison of our work with Bellotti's paper [7] where the same technique has been applied on a dataset largely inferior in size with respect to ours; it emerges that our system reaches a significantly higher sensitivity at comparable FPs rates. This result has probably to be attributed to the MSM based qualitatively superior method of ROI detection and segmentation we developed.

Fig. 17 presents as dashed line the FROC curve of the system's performance without using the double-threshold cut. The aforementioned curve has been obtained by using the same neural network architecture (7-3-1) and the same training technique, while the patterns used during this case's training phase were $148 \mathrm{TP}$ and $1332 \mathrm{FP}$. From the visual comparison of the two curves, it is easy to notice the CAD system improvement due to the two-threshold cut: at $2.5 \mathrm{FP} / \mathrm{scan}$, the sensitivity increases from $79 \%$ to about $88 \%$, that is a sensitivity improvement of about $9 \%$ at a fixed rate of false positives, which constitutes a fair amount. A potential bias introduced by the double-threshold cut should range definitely below this increase. The performances reached without the double-threshold cut are still comparable to the ones of the two alternative systems validated on the same database. Moreover, the classifier's ability to distinguish between healthy and pathologic classes, despite the highly increased complexity of the classification problem, is another proof of the proposed segmentation method effectiveness: the accuracy of segmentation obviously leads to better separation in the features space.

\section{Conclusions}

The characteristics of slices sequence analysis make the nodule detection in lung CT scans a hard task for the humans involved in their analysis. Therefore, CAD systems can be a helpful tool for the radiologists in lung cancer diagnosis.

We developed an advanced computerized method for the automatic detection of internal and juxtapleural nodules on low-dose and thin-slice lung CT scans. This method consists of an initial selection of nodule candidates list followed by the segmentation of each nodule candidate and the classification of the features computed for each segmented nodule candidate. We propose a new lung nodules 3D segmentation technique based on deformable MSMs for images from CT datasets. The result is a robust and efficient segmentation process able to converge, identifying the shape of the generic ROI, after a few iterations. This method has been validated on the 148 nodules with diameters superior to $3 \mathrm{~mm}$ contained in $84 \mathrm{CT}$ scans. The best performance of our CAD is achieved at a sensitivity of $88 \%$ for the nodules at a corresponding FP rate of $2.5 \mathrm{FPs} / \mathrm{CT}$. In this case, the combination of the segmentation procedure with the neural classifier is able to reject about $94 \%$ of the FP generated by the ROI hunter. Our encouraging results show that the use of the 3D AC model and the FPs reduction process based on feature analysis constitutes a valid approach to the segmentation and the classification of lung nodules.

In a future paper we will focus on the results of the testing of the proposed approach on more datasets. For a better estimation of the accuracy and limitations of the proposed system, we are currently engaged in collecting more data from 100 additional patients with different types of pulmonary nodules, data on which our system will be tested. We will also investigate the possibility to add other geometrical features describing the shape of malignant and benign nodules in order to determine whether this will lead to more accurate diagnosis with respect the current methodology.

\section{Conflict of interest statement}

None declared.

\section{References}

[1] A. Jemal, T. Murray, E. Ward, A. Samuels, R.C. Tiwari, A. Ghafoor, E.J. Feuer M.J. Thun, Cancer statistics, CA Cancer J. Clin. 55 (2005) 10-30.

[2] A. Micheli, P. Baili, M. Quinn, E. Mugno, R. Capocaccia, P. Grosclaude, Life expectancy and Cancer Survival, The EUROCARE Working Group, in the EUROCARE-3 cancer registry areas, Ann. Oncol. 14(5) (2003) V28-V40.

[3] G.K. Singh, B.A. Miller, B.F. Hankey, Changing area socioeconomic patterns in U.S.cancer mortality, 1950-1988. Part II: lung and colorectal cancers, J. Natl. Cancer Inst. 94 (2002) 916-925.

[4] D.C. Ihde, J.D. Minna, Non-small cell lung cancer. Part I: biology, diagnosis, and staging, Curr. Probl. Cancer 15 (2) (1991) 61-104.

[5] J.P. Ko, M. Betke, Chest CT: automated nodule detection and assessment of change over time-preliminary experience, Radiology 218 (2001) 267-273.

[6] S.G. Armato III, M.L. Giger, H. MacMahon, Automated detection of lung nodules in CT scans: preliminary results, Med. Phys. 28 (2001) 1552-1561.

[7] R. Bellotti, et al., A CAD system for nodule detection in lung CTs based region growing and active contour models, Med. Phys. 34 (2007) 4901-4910.

[8] M.L. Giger, K.T. Bae, H. MacMahon, Computerized detection of pulmonary nodules in computed tomography images, Invest. Radiol. 29 (1994) 459-465.

[9] W. Kostis, A. Reeves, D. Yankelevitz, C. Henschke, Three dimensional segmentation and growth-rate estimation of small pulmonary nodules in helical CT images, IEEE Trans. Med. Imaging 22 (10) (2003) 1259-1274.

[10] M.S. Brown, J.G. Goldin, S. Rogers, H.J. Kim, R.D. Suh, M.F. McNitt-Gray, S.K. Shah, D. Truong, K. Brown, J.W. Sayre, D.W. Gjertson, P. Batra, D.R. Aberle, Computer-aided lung nodule detection in ct: results of large-scale observer test, Acad. Radiol. 12 (2005) 681-686.

[11] A.S. Roy, S.G. Armato III, A. Wilson, K. Drukker, Automated detection of lung nodules in CT scans: false positives reduction with the radial-gradient index, Med. Phys. 33 (2006) 1133-1140.

[12] J. Pu, et al., An automated CT based lung nodule detection scheme using geometric analysis of signed distance field, Med. Phys. 35 (2008) 3453-3462.

[13] L. Fan, J. Qian, B.L. Odry, H. Shen, D. Naidich, G. Kohl, E. Klotz, Automatic segmentation of pulmonary nodules by using dynamic 3-D cross correlation for interactive CAD systems, in: Proceedings of the SPIE Medical Imaging, vol. 4684, 2002, pp. 1362-1369.

[14] K. Okada, D. Comaniciu, A. Krishnan, Robust anisotropic gaussian fitting for volumetric characterization of pulmonary nodules in multislice CT, IEEE Trans. Med. Imaging 24 (3) (2005) 409-423.

[15] Z. Ge, B. Sahiner, H.-P. Chan, L.M. Hadjiiski, J. Wei, N. Bogot, P.N. Cascade, E.A. Kazerooni, C. Zhou, Computer aided detection of lung nodules: false positive reduction using a 3-D gradient field method, in: Proceedings of the Medical Imaging 2004: Image Processing, San Diego, CA, 5370-110, 2004.

[16] K. Suzuki, S.G. Armato III, F. Li, S. Sone, K. Doi, Massive training artificial neural network (MTANN)for reduction of lung nodules in low dose computed tomography, Med. Phys. 30 (2003) 1602-1617.

[17] G. Ravenel, E.M. Scalzetti, W. Huda, W. Garrisi, Radiation exposure and image quality in chest CT examinations, AJR, Am. J. Roentgenol. 177 (2001) 279-284.

[18] R. Tangaro, Bellotti, F. De Carlo, et al., MAGIC-5: An Italian mammographic database of digitized images for research, La Radiologia Medica, vol. 113(4), 2008, pp. 477-485.

[19] S.G. Armato III, et al., Lung image database consortium: developing a resource for the medical imaging research community, Radiology 232 (2004) 739-748.

[20] M.F. McNitt-Gray, et al., The lung image database consortium (LIDC) data collection process for nodule detection and annotation. Acad. Radiol. 14 (2007) 1464-1474.

[21] L. Dornheim, K.D. Tnnies, J. Dornheim, Stable dynamic 3D shape models, in: Proceedings of IEEE International Conference on Image Processing (ICIP), 3, 1530632, 1276-1279, 2005.

[22] Dornheim Jana, Seim Heiko, Preim Bernhard, et al., Segmentation of neck lymph nodes in CT datasets with stable 3D mass-spring models: segmentation of neck lymph nodes, Acad. Radiol. 14 (11) (2007) 1389-1399.

[23] A. Goshtasby, D.A. Turner, L.V. Ackerman, Matching of tomographic slices for interpolation, IEEE Trans. Med. Imaging 11 (1992) 507-516.

[24] T.Y. Lee, W.H. Wang, Morphology-based three-dimensional interpolation, IEEE Trans. Med. Imaging 19 (2000) 711-721.

[25] Q. Li, S. Katsuragawa, K. Doi, Computer aided diagnostic scheme for lung nodule detection in digital chest radiographs by use of a multiple template matching technique, Med. Phys. 28 (2001) 2070-2076.

[26] T.W. Ridler, S. Calvard, Picture thresholding using an iterative selection method, IEEE Trans. Syst. Man Cybern. 8 (1978) 630-632.

[27] D. Cascio, et al., Automated detection of lung nodules in low-dose computed tomography, Comput. Assist. Radiol. Surg. 2 (2007) 351-372.

[28] D. Terzopoulos, K. Fleischer, Deformable models, Vis. Comput. 4 (6) (1988) 306-331.

[29] D. Terzopoulos, A. Witkin, M. Kass, Constraints on deformable models: recovering 3D shape and nonrigid motion, Artif. Intell. 36 (1) (1988) 91-124. 
[30] G.L. Masala, S. Tangaro, B. Golosio, et al., Comparative study of feature classification methods for mass lesion recognition in digitized mammograms, Nuovo Cimento: C: Geophys. Space Phys. 30 (2007) 305-3016.

[31] G.L. Masala, B. Golosio, P. Oliva, et al., Classifiers trained on dissimilarity representation of medical pattern: a comparative study, Nuovo Cimento: C: Geophys. Space Phys. 28 (2005) 905-912.

[32] F. Fauci, S. Bagnasco, R. Bellotti, et al., Mammogram segmentation by contour searching and massive lesion classification with neural network, in: IEEE Nuclear Science Symposium Conference Record, vol. 5, 2004, pp. 2695-2699.

[33] F. Fauci, D. Cascio, A. La Manna, et al., A fourier-based algorithm for microcalcification enhancement in mammographic images, in: IEEE Nuclear Science Symposium Conference Record, Article Number 4774254, 2008, pp. 4388-4391.
[34] L. Vivona, D. Cascio, R. Magro, et al., A fuzzy logic C-means clustering algorithm to enhance microcalcifications clusters in digital mammograms, in: IEEE Nuclear Science Symposium Conference Record, Article Number 6162551, 2012, pp. 3048-3050.

[35] M. Stone, Cross-validatory choice and assessment of statistical predictions, J. R. Stat. Soc. Ser. B Methodol. 36 (1974) 111-147.

[36] Bruno Golosio, et al., A novel multithreshold method for nodule detection in lung CT, Med. Phys. 36 (8) (2009) 3607-3618.

[37] Temesguen Messay, et al., A new computationally efficient CAD system for pulmonary nodule detection in CT imagery, Med. Image Anal. 14 (3) (2010) 390-406. 